\title{
Cystic Fibrosis Mice Lacking Muc1 Have Reduced Amounts Of Intestinal Mucus
}

Robin R. Parmley and Sandra J. Gendler

Mayo Clinic Scottsdale, Department of Biochemistry and Molecular Biology, Scottsdale, Arizona 85259

\begin{abstract}
Normally a thin layer of mucus covers the surface of the gastrointestinal tract protecting the epithelial cells from their environment. In cystic fibrosis (CF), mucus accumulation is abnormally high, resulting in severe intestinal obstruction. The major structural components of mucus are large mucin glycoproteins. We determined specific mucin RNA and protein expression in the gastrointestinal tract of inbred CF transmembrane conductance regulator (CFTR) knockout (CF) mice and correlated expression with histological analyses of tissues. Mucins were detected histochemically using general carbohydrate stains and specific mucin antibodies. Mucin RNA levels were determined by reverse transcription-PCR. Comparisons were made between CF mice and control siblings, all maintained on a liquid diet after weaning. Analyses of the mucins Muc2, Muc3, and Muc5ac showed lower levels of RNA expression in the CF mice and similar levels of protein. Significantly, there was a sixfold increase in Muc1 RNA expression in the colon of the CF mouse and a moderate increase in Muc1 protein. Further, CF mice lacking Muc1 exhibited greatly diminished intestinal mucus obstruction when compared with Muc1expressing CF mice and had better survival on solid food. We suggest that Muc1 plays an important role in the mucus obstructions observed in the gastrointestinal tract of the CFTR knockout mouse. (J. Clin. Invest. 1998. 102:17981806.) Key words: meconium ileus - gastrointestinal tract • reverse transcription-polymerase chain reaction $\bullet$ immunohistochemistry $\bullet$ mouse cystic fibrosis model
\end{abstract}

\section{Introduction}

The luminal surface of the tracheobronchial, gastrointestinal (GI), ${ }^{1}$ and reproductive tracts are lubricated and protected by the production of a thin mucus barrier. In its normal state the

Address correspondence to Sandra J. Gendler, Mayo Clinic Scottsdale, Department of Biochemistry and Molecular Biology, S.C. Johnson Research Building, 13400 East Shea Blvd., Scottsdale, AZ 85259. Phone: 602-301-7137; FAX: 602-301-7017; E-mail: gendler. sandra@mayo.edu

Received for publication 24 April 1998 and accepted in revised form 24 September 1998.

1. Abbreviations used in this paper: $\mathrm{CF}$, cystic fibrosis; CFTR, CF transmembrane conductance regulator; GI, gastrointestinal; Muc, mouse mucin; MUC, human mucin; RT, reverse transcription.

J. Clin. Invest.

(C) The American Society for Clinical Investigation, Inc. 0021-9738/98/11/1798/09 \$2.00

Volume 102, Number 10, November 1998, 1798-1806

http://www.jci.org mucus is composed of $95 \%$ water, $2 \%$ glycoproteins, and $1 \%$ each of protein, lipids, and inorganic salts (1). The major structural components of mucus, which confer its viscoelastic properties, are glycoproteins called mucins. Mucins are classified as one of two main forms: membrane-associated or secreted. In general, a mucin subunit consists of a peptide core, making up $20 \%$ of the molecule, with diverse carbohydrate side chains comprising the remaining $80 \%$. The side chains typically are highly sulfated and terminate in sialic acid. The size of a mucin subunit can range from 200 to $1,000 \mathrm{kD}$. The subunits of secreted mucins, via end-to-end disulfide bonding, form large oligomers that thinly coat the epithelial surface (2). In the intestinal epithelia and in the lower airways the goblet cell is a major producer of secreted mucins. Goblet cells can release large quantities of mucins in milliseconds in defense against acute insult. In response to chronic insult, they can increase in numbers leading to hypersecretion of mucus. The most serious complication in cystic fibrosis (CF) is the retention of large amounts of abnormally viscous mucus in the airways and in the GI tract. Mucin is secreted from intracellular granules via two mechanisms: a constitutive pathway and a regulated pathway. The constitutive pathway involves continuous secretion of a small amount of mucin necessary for maintaining protection and lubrication of the epithelial surface. In the regulated pathway, mucin release is triggered by various environmental stimuli (3). In disease states such as CF, which are severely complicated by mucus accumulation, it is crucial to understand the mechanisms of secretion and accumulation. General methods of measuring mucus secretion involving radiolabeled precursors, lectin stains, and "mucus" stains (i.e., Alcian blue and periodic acid-Schiff [PAS]) suggested that increased amounts of mucins play an important role in the pathology of CF. With recent advances in the field of mucin biology it is now possible to look at specific mucin production. Nine human mucin genes and five rodent genes have been cloned or partially cloned to date, and thus antibodies and molecular probes have made it possible to look at message levels and protein expression of the specific molecules.

Our studies have focused on a mouse model of CF that was developed by Koller and colleagues (4). Due to the introduction of a stop codon in exon 10, the CF transmembrane conductance regulator (CFTR) gene gives rise to a truncated, nonfunctional gene product. Homozygous CFTR knockout mice $\left(\mathrm{Cftr}^{\mathrm{m} 1 \mathrm{UNC}} / \mathrm{Cftr}^{\mathrm{m} 1 \mathrm{UNC}}\right)$ have severe intestinal obstruction that commonly results in death at weaning. Although a unifying pathological feature of CF in humans is obstruction of the respiratory, reproductive, and GI tracts, mucus accumulation and obstruction in pathogen-free CF mice has been observed only in the GI tract (5). In humans, as many as $10-20 \%$ of CF infants are diagnosed with meconium ileus and an even greater percentage of CF adolescents and adults have distal intestinal obstruction syndrome, which is a severe recurring condition (6). Although rarely life threatening in humans, relief from these intestinal obstructions most often involves unpleasant medical treatment or surgical intervention. A full understand- 
ing of the pathogenesis of mucus accumulation in the CF intestine awaits clarification of the glycoconjugate (mucin) content of these secretions. This study was designed to investigate the possible upregulation in production of a specific mucin(s) in the GI tract of the CF mouse.

The molecules that we have studied are Muc1, Muc2, Muc3 (formerly designated M2/rMuc176), and Muc5ac. (Rodent mucins are designated as Muc and human mucins as MUC.) Muc1 is a transmembrane protein that is expressed by most simple secretory epithelia (7). The core protein consists of an $\mathrm{NH}_{2}$ terminal signal sequence, a tandem repeat domain containing numerous serines and threonines, a hydrophobic transmembrane domain, and a cytoplasmic domain $(8,9)$. The existence of a secreted form of MUC1, lacking the cytoplasmic tail, has also been determined (10-15). In the small intestine and colon, Muc1 is produced at very low levels by enterocytes. Muc2 and Muc5ac are secreted glycoproteins, whereas Muc3 has been described recently as a transmembrane mucin (16-19). In addition to numerous tandem repeats, these mucins have cysteinerich domains that are responsible for self-dimerization. The secreted mucins are much larger than the transmembrane Muc1, reaching sizes of $1,000 \mathrm{kD}$.

Initial experiments in this study showed a large increase in luminal mucus in the colon and ileum of the CF mice. When specific mucins were studied using reverse transcription (RT)PCR, immunohistochemistry, and knockout breeding strategies, it was surprising that Muc1, a transmembrane mucin, appeared to play a significant role in the fatal obstructions observed in the GI tract of the CF mouse.

\section{Methods}

Animals. Mice heterozygous for the S489X mutation (4) were a generous gift from Dr. Beverly Koller (University of North Carolina, Chapel Hill, NC). The heterozygous mice were subsequently crossed to generate mice that were homozygous for the mutation (CF mice). The animals were housed in microisolator cages on celludry/carefresh (60:40) bedding in a specific pathogen-free facility. The pups were weaned at $24 \mathrm{~d}$ of age at which time samples of tail DNA were taken and the animals were genotyped by PCR. Mice identified as CF mice ( $\left.\mathrm{Cftr}^{\mathrm{m} 1 \mathrm{UNC}} / \mathrm{Cftr}^{\mathrm{m} 1 \mathrm{UNC}}\right)$ were weaned on to a liquid mouse diet (product \#F3017; BioServ, Frenchtown, NJ) that was prepared daily as suggested by the manufacturer. The control mice (heterozygous $\mathrm{Cftr}^{\mathrm{m} 1 \mathrm{UNC}}$ and wild-type C57Bl/6) were maintained on the same liquid diet. Sterile water was provided for all animals. All of the mice in this study were either fourth or eighth generation $\mathrm{C} 57 \mathrm{Bl} / 6$ and both sexes were equally represented. There were no significant differences in mucin expression among the groups studied $\left(\mathrm{Cftr}^{\mathrm{m} 1 \mathrm{UNC}} / \mathrm{Cftr}^{\mathrm{m} 1 \mathrm{UNC}}\right.$, heterozygous $\mathrm{Cftr}^{\mathrm{m} 1 \mathrm{UNC}}$, and wild-type $\mathrm{C} 57 \mathrm{Bl} / 6$ ) on the basis of generation or gender. The ages of the mice studied ranged from 3.5 to 25 wk. In each experiment the weanlings $(3.5 \mathrm{wk}, n=4)$ were analyzed both independently and with the rest of the adult mice.

Mice heterozygous for the S489X mutation were also mated with Muc1 knockout mice (20) in generation of C57B1/6 mice with CF that lacked Muc1 (Muc1-deficient CF mice). At $24 \mathrm{~d}$ of age, some of these mice were weaned on to the liquid diet and others on to solid food. The mice were maintained as described above.

Tissues. Mice were killed by $\mathrm{CO}_{2}$ asphyxiation and GI tissues were removed for RNA preparation and for histological analysis. The stomach, ileum, cecum, colon, and liver were taken for these purposes. To ensure uniformity, the entire stomach, cecum, colon, and 4 $\mathrm{cm}$ of ileum just proximal to the ileocecal sphincter were removed. A small portion of liver was taken as a negative control. All tissues upon removal were rinsed in PBS and either snap-frozen in liquid nitrogen for RNA preparation or methacarn-fixed ( $60 \%$ methanol, $30 \%$ chloroform, $10 \%$ acetic acid) for histological studies.

Amplified genes. Muc1 is expressed by most simple secretory epithelia. Although most commonly known as a cell-associated mucin, a secreted form of human MUC1 has been described (10-15). Muc2, a large secreted mucin, is a goblet cell product that is found mainly in the small intestine and colon (17). Muc3 is another intestinal mucin that has been cloned partially by two different groups of investigators $(18,19)$. Probes for M2 and for rMuc176 both hybridize to a 9-kb RNA from small intestine and colon. It is believed that M2 and rMuc176 represent different regions of the same intestinal mucin gene (17). Recent data suggested that M2 and rMuc176 are part of Muc3, which has been described as a possible transmembrane mucin (19). Muc5ac is classified as a tracheobronchial mucin; however, it is also highly expressed in the mucous cells of the stomach and by the goblet cells of the small intestine and colon (20). (Mucin genes are reviewed in reference 2.)

$R N A$ extraction and analysis by RT-PCR. Since the peptide core of mucins consists of a variable number of tandem repeats and the RNA encoding it is very large (2), upon isolation the RNA is commonly degraded and thus represented by a large smear on Northern analysis. For this reason, RT-PCR was the method chosen for quantitation of mucin RNA levels in these studies. Total RNA was extracted from each tissue using TRIzol ${ }^{\circledR}$ reagent (GIBCO BRL, Grand Island, NY) as recommended by the manufacturer. RNA pellets were resuspended in sterile water and concentrations were determined by absorbance at $260 \mathrm{~nm}$. The samples were subsequently stored at $-70^{\circ} \mathrm{C}$ until use. A $10-\mu \mathrm{g}$ sample of total RNA in a total volume of $62.5 \mu \mathrm{l}$ was DNase treated with $50 \mathrm{U}$ of RNase-free DNase (Boehringer Mannheim, Indianapolis, IN) at $37^{\circ} \mathrm{C}$ for $15 \mathrm{~min}$ followed by denaturation in $0.05 \mathrm{M}$ Tris- $\mathrm{HCl}, \mathrm{pH} 7.5$, at $94^{\circ} \mathrm{C}$ for $2 \mathrm{~min}$. The samples were reverse-transcribed with $200 \mathrm{U}$ of Moloney murine leukemia virus reverse transcriptase (GIBCO BRL), 100 pmol random hexanucleotide primers, 100 pmol dT oligomers, and $16 \mathrm{mM}$ dNTP (Pharmacia Biotech, Uppsala, Sweden) in a total volume of $125 \mu \mathrm{l}$ at $42^{\circ} \mathrm{C}$ for $1 \mathrm{~h}$. The cDNA was aliquoted and stored at $-20^{\circ} \mathrm{C}$. RT for Muc2 was as described above; however, 25 pmol of $3^{\prime}$ primer was used in place of the random hexanucleotides and dT oligomers. Muc1, Muc2, Muc3, and actin sequences were subsequently amplified by PCR using $12.5 \mu \mathrm{l} \mathrm{cDNA}$ in a $50-\mu \mathrm{l}$ reaction volume. Due to the increased expression levels of Muc5ac, $2 \mu \mathrm{l}$ of cDNA was used in a $50-\mu 1$ reaction. PCR was performed using $2.5 \mathrm{U}$ of Taq polymerase (Boehringer Mannheim), $25 \mathrm{pmol}$ of sense and antisense primers, and $1 \mu \mathrm{l}$ of formamide. All sense primers were $5^{\prime}$ labeled with phosphoramidite dyes (Applied Biosystems, Division of Perkin Elmer, Foster City, CA). Each set of primers was optimized for annealing temperature and titrated with respect to cycle number. For each mucin and for actin a cycle number that fell in the exponential portion of the titration curve was used in order to obtain accurate quantitation. The primers for each gene and their PCR conditions are shown in Table I.

The primers were designed to include the restriction sites listed in Table I and thus correct PCR products were confirmed by restriction analysis. The fluorescent PCR products $(1 \mu \mathrm{l})$ were separated by capillary electrophoresis in 3\% acrylamide containing 6.6 M urea (Prism 310 Genetic Analyzer; Applied Biosystems). Band densities were assessed using automated laser densitometry and Genescan software (Applied Biosystems). The relative mucin expression levels in each sample were calculated using $\beta$-actin levels as a reference. Furthermore, actin levels among different groups of animals and also among different tissues were similar, with average expression levels falling within the $95 \%$ confidence interval.

Immunohistochemistry. Methacarn-fixed tissues were embedded in paraffin blocks and sectioned to a thickness of $5 \mu \mathrm{m}$. General mucin stains used were Alcian blue and PAS. The two stains detect acidic carbohydrate moieties and vicinal hydroxyl groups, respectively (22). Muc1 protein was detected using CT1, a polyclonal antiserum raised to a synthetic peptide in the cytoplasmic tail of MUC1 (23). Muc2 was detected using PH497, a polyclonal antiserum made 


\begin{tabular}{|c|c|c|c|c|c|c|c|}
\hline Gene & s/as & Primer sequence $\left(5^{\prime}\right.$ to $\left.3^{\prime}\right)$ & $\begin{array}{l}\text { Annealing } \\
\text { temperature }\end{array}$ & Cycle No. & $\begin{array}{l}\text { cDNA } \\
\text { location }\end{array}$ & $\begin{array}{c}\text { Restriction } \\
\text { enzyme }\end{array}$ & Ref. \\
\hline & & & & & $b p$ & & \\
\hline \multirow[t]{2}{*}{ Muc1 } & $\mathrm{s}$ & CCCCAGTTGTCTGTTGGGGTC & $62^{\circ} \mathrm{C}$ & 25 & $921-1275$ & PstI & 9 \\
\hline & as & GGATTCTACCACCACGGAGCC & & & & & \\
\hline \multirow[t]{2}{*}{ Muc2 } & $\mathrm{s}$ & GGGACCCACACTTTGTCACC & $58^{\circ} \mathrm{C}$ & 17 & $439-657$ & AvaII & 16 \\
\hline & as & GGGCATCATGTGCACAGTCTTG & & & & & \\
\hline Muc3 & $\mathrm{s}$ & CCATCTGTCTTCACC & $46^{\circ} \mathrm{C}$ & 25 & $581-793$ & HinfI & 17 \\
\hline (M2/rMuc176) & as & TGAAGATGGGGATAC & & & & & \\
\hline \multirow[t]{2}{*}{ Muc5ac } & $\mathrm{s}$ & GGACCAAGTGGTTTGACACTGAC & $67^{\circ} \mathrm{C}$ & 28 & $854-1111$ & DdeI & 19 \\
\hline & as & CCTCATAGTTGAGGCACATCCCAG & & & & & \\
\hline \multirow[t]{2}{*}{ Actin } & $\mathrm{s}$ & GTGGGCCGCTCTAGGCACCA & $62^{\circ} \mathrm{C}$ & 15 & $25-265$ & Bg1II & 21 \\
\hline & as & TGGCCTTAGGGTGCAGGGGG & & & & & \\
\hline
\end{tabular}

$s$, sense strand primer; as, antisense strand primer. Restriction enzymes were used to verify the correct PCR product. M2/rMuc176 are partial clones of the same gene as Muc3. The reference refers to where the sequence was obtained.

to the deglycosylated core of rat Muc2 (24). PH497 was a generous gift from Dr. Gunnar Hansson (Göteborg University, Göteborg, Sweden). Muc3 protein was detected using a polyclonal antiserum (RIB-3) to the deglycosylated core of rMuc176 (18). RIB-3 was a generous gift from Drs. Young Kim and Jim Gum (University of California at San Francisco, San Francisco, CA). Antiserum to native Muc5ac (HO1) was a generous gift from Dr. Sam Ho (University of Minnesota, Minneapolis, MN) (19). Before staining, the tissues were cleared in xylene and rehydrated using a series of decreasing ethanol concentrations. The slides were then blocked with $50 \%$ FCS in PBS for $1.5 \mathrm{~h}$ at $25^{\circ} \mathrm{C}$. The tissues were subsequently incubated at $25^{\circ} \mathrm{C}$ for $1.5 \mathrm{~h}$ with an appropriate dilution of primary antiserum. After a series of three 5-min washes in PBS, the samples were incubated at $25^{\circ} \mathrm{C}$ for $1.5 \mathrm{~h}$ with a secondary antibody conjugated to Cyanin-3. The tissues were again washed as described above and coverslips were applied using Gel/Mount (Biomeda, Foster City, CA). Staining was observed and photographed with a Nikon Microphot-FXA.

Statistical analysis. Differences in mean mucin expression levels between the $\mathrm{CF}$ mice and the control mice were assessed pair-wise based on Duncan's multiple range test. The criterion for statistical significance was set at $P<0.05$.

\section{Results}

Mucus accumulation in the intestinal tract of CF mice. Fig. 1 shows the dramatic increase in PAS-positive material (most likely mucus) in the intestinal tissue of two CF mice. Both the ileum and colon of the sibling control mice showed a normal PAS staining pattern with a thin layer of mucus covering the epithelial cells along the lumen (Fig. 1, $A$ and $C$ ). However, the lumens of both intestinal tissues in the CF mice were packed with mucus (Fig. 1, $B$ and $D$ ). It is this accumulation of material that is most likely causing the blockages that are so prevalent in the intestinal tract of the CF mouse. Comparisons of goblet cell staining that were made at $50-\mu \mathrm{m}$ levels along the villi from tip to base were similar in both sets of mice (not shown). However, as shown by the arrowheads in Fig. 1, the goblet cells in the control mice routinely stained more intensely and appeared distended as if they contained greater amounts of stored mucins. Similar observations were noted with Alcian blue staining in the colon and ileum of the same set of mice (not shown).
Muc1 message and protein levels in CF mice. Mucin expression was studied in the colon, ileum, cecum, and stomach of the CF mice and their sibling controls. Muc1 RNA expression was observed at low levels in the mouse colon and ileum, and at high levels in the stomach (Fig. 2 A). Surprisingly, Muc1 RNA expression in the colon was significantly increased by sixfold in the CF mice when compared with littermate controls. When only the 3.5-wk-old weanlings were compared,

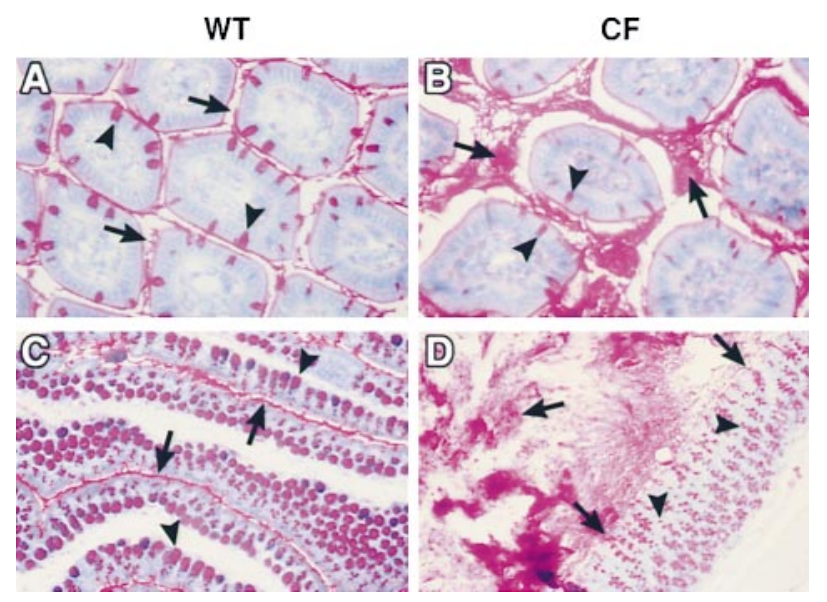

Figure 1. Detection of intestinal mucus by PAS in CF and control mice. $(A)$ Ileum of a control mouse in which intestinal villi were lined with a thin layer of mucus (arrows) and mucin-containing goblet cells appeared very distended (arrowheads). (B) Ileum of a CF mouse showing dilated crypts filled with PAS-positive mucus (arrows). The goblet cells (arrowheads) did not appear as distended as those in the control intestine. $(C)$ Colon of a control mouse showing a thin layer of mucus covering the epithelial surface (arrows) and goblet cells lining the crypts (arrowheads). (D) Colon of a CF mouse where mucincontaining goblet cells are visible yet appear much less distended ( $\mathrm{ar}$ rowheads) than those of the control mouse in $C$. The intestinal lumen of the CF mouse is full of mucus (arrows) that is streaming out of the goblet cells. $A$ and $B$ are ileal cross-sections at a magnification of 400 . $C$ and $D$ are sagittal sections of the colon magnified 100 -fold. All tissues shown are from animals that were 13 wk of age. These data are representative of four sets of PAS staining. 
A
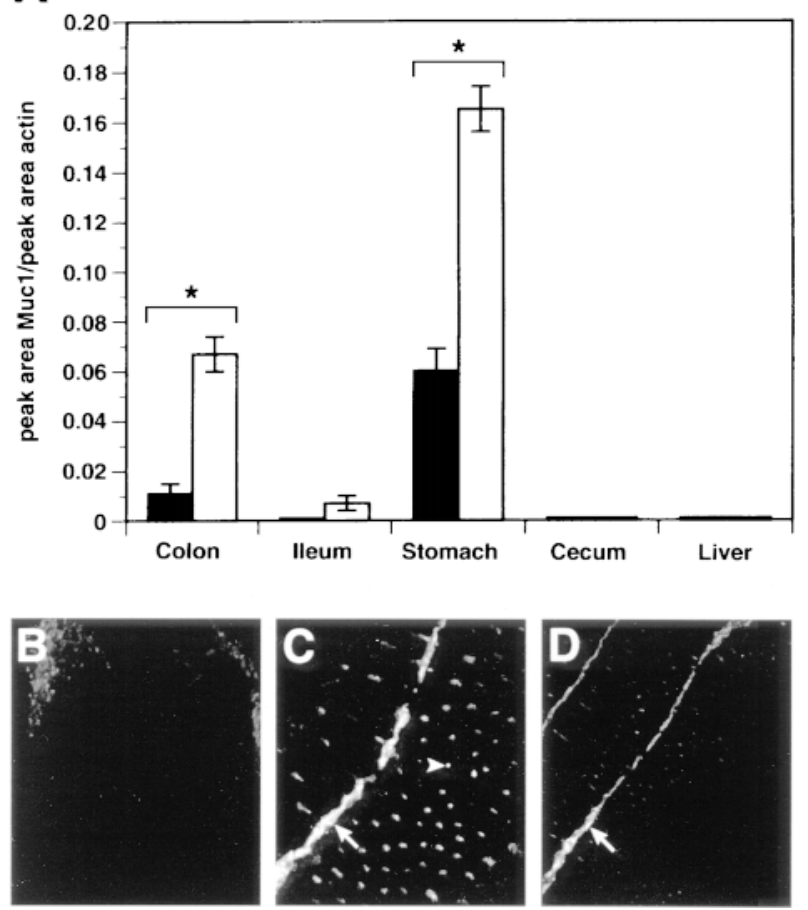

Figure 2. Muc1 expression in GI tissues of CF mice. $(A)$ Expression of Muc1 RNA in GI tissues of CF and control mice. Muc1 RNA levels were determined in the colon, ileum, cecum, stomach, and liver of $14 \mathrm{CF}$ mice (open bars) and 7 control mice (filled bars). Error bars represent SEM. *Significant RNA expression differences were found in the colon and in the stomach between the control and the CF mice. $(B-D)$ Immunohistochemical micrographs of $\mathrm{CF}$ and control colon using polyclonal antiserum (CT1) that was generated to a cytoplasmic tail peptide of MUC1. $(B)$ Colon of a 13-wk-old control mouse where expression of Muc1 was not visible. (C) Colon of a 13-wk-old CF mouse showing Muc1 staining in the intestinal epithelium (arrowhead). This staining pattern was also observed in two additional CF mice; however, no Muc1 protein was detected in nine other CF colons stained with CT1. $(D)$ Colonic staining was blocked by the immunizing peptide. Arrows designate nonspecific luminal staining. All panels are shown $\times 100$. Exposure times and camera settings for photographing were identical for all panels.

however, there were no differences in Muc1 expression (not shown). Thus, it appears that increased colonic Muc1 RNA expression developed as the animals aged and did not exist at weaning. In addition, gastric Muc1 expression levels were significantly higher (2.8-fold) in the CF mice than in control mice, suggesting an increase in expression in this $\mathrm{CF}$ tissue as well.

Due to the low levels of Muc1 protein expression in the colon, antibody staining in this tissue was difficult to detect. However, in $25 \%$ (3/12) of the sibling pairs studied, histological analyses using CT1, an antiserum to the cytoplasmic tail of Muc1, revealed a moderate increase in Muc1 protein in the colon of the CF mouse. Muc1 protein was not observed in the normal colon (Fig. $2 \mathrm{~B}$ ). In a portion of the CF mice, however, some of the epithelial cells lining the intestinal crypts stained positive, revealing Muc1 protein expression (Fig. 2C). This expression was specific for Muc1, as peptide to which the antiserum was originally made was able to inhibit staining (Fig. $2 D$ ). It was not possible to detect increases in secreted Muc1 in the $\mathrm{CF}$ lumen as antibodies to the glycosylated, extracellular re- gion of the molecule do not exist for the mouse. The streaks of fluorescence observed in the lumen are background as the CT1 peptide did not inhibit this staining. Several lectins were also studied with the hopes of finding one specific for Muc1, but this proved unsuccessful. There was no obvious increase in Muc1 antibody staining in the ileum or stomach of the CF mice (not shown).

Muc2, Muc3, and Muc5ac expression in CF mice. All of the secreted mucins studied showed a significant decrease in RNA
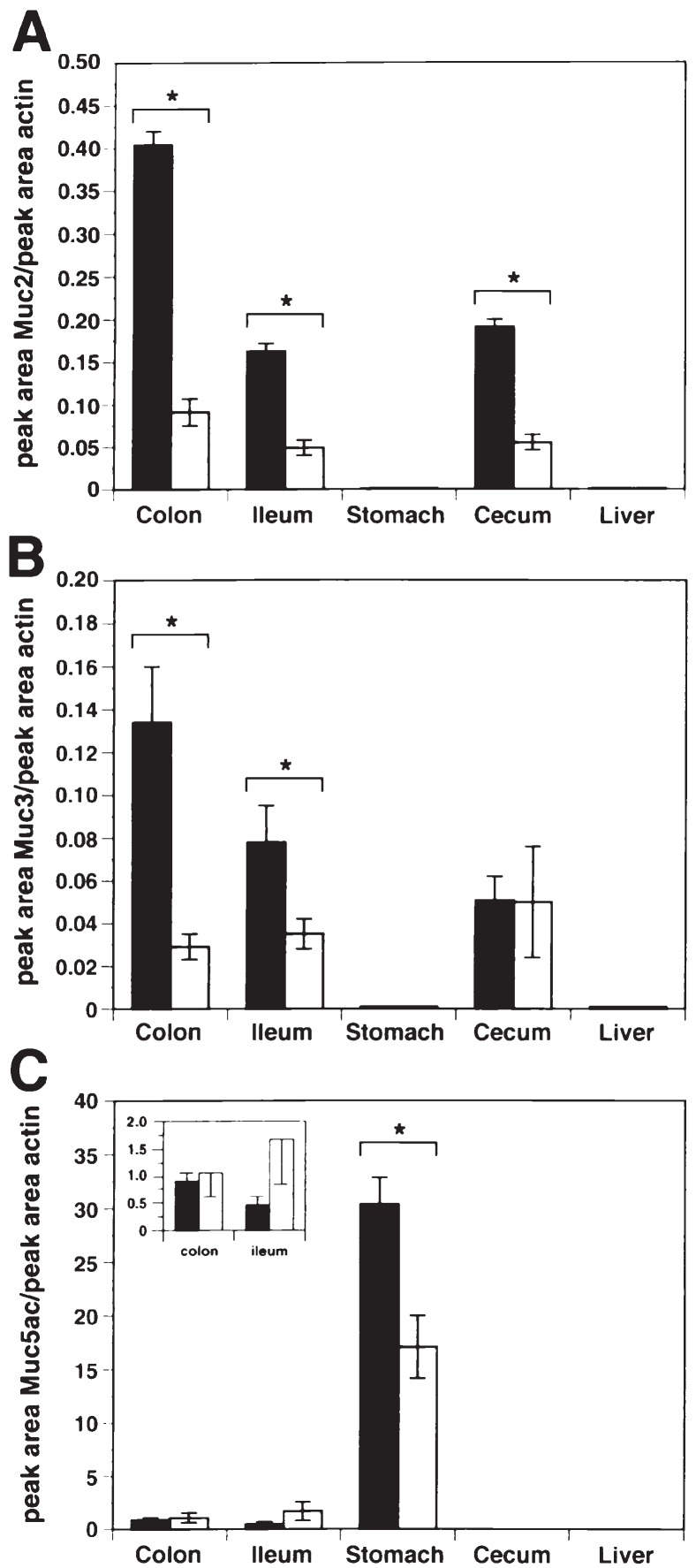

Figure 3. RNA expression of secreted mucins in the GI tissues of $\mathrm{CF}$ mice. RNA levels for Muc2 $(A), \operatorname{Muc3}(B)$, and Muc5ac $(C)$ were determined in the colon, ileum, cecum, stomach, and liver of $14 \mathrm{CF}$ mice (open bars) and 7 control mice (filled bars). Asterisks denote statistical significance. 
expression in the $\mathrm{CF}$ mice as compared with their littermate controls (Fig. 3, $A-C$ ). This decrease in mucin expression was observed in the colon and in the ileum of the CF mice with Muc2 and Muc3, in the cecum with Muc2, and in the stomach with Muc5ac.

Histological analyses of the GI tissues using antisera to Muc2 and Muc3 (rMuc176) revealed no obvious differences in protein expression between the $\mathrm{CF}$ mice and their controls. Fig. 4, $A$ and $B$, shows the cellular expression pattern of Muc2 protein in the ileum of a control mouse and a CF mouse, respectively. As expected, the Muc2 antiserum was a nice goblet cell marker but did not recognize the glycosylated molecule. Intracellular expression of immature Muc2 protein appeared similar between the CF mouse and the control with both tissues showing strong staining in the crypts and moderate staining of the goblet cells lining the villi. A preimmune antiserum used to stain the same sections of tissue was consistently negative with respect to the goblet cells; however, the faint staining of the luminal contents was positive in the preimmune control and therefore considered as nonspecific background (not shown). The staining pattern for Muc3 was very similar to that of Muc2, with antiserum against nonglycosylated rMuc176 recognizing the mucin within the goblet cells of the ileum (Fig. 4, $D$ and $E$ ). There was a slight decrease in goblet cell staining in the CF animals (Fig. $4 E$ ) compared with the wild-type control animals (Fig. $4 \mathrm{D}$ ). With the preimmune control antiserum there was no staining of the goblet cells and faint staining of the luminal contents (not shown). However, the staining of the luminal contents in the $\mathrm{CF}$ mouse was noticeably brighter with the rMuc176 (Muc3) antiserum than with its control antiserum, suggesting that some of the staining could be specific for rMuc176. The antiserum raised to native Muc5ac appeared to recognize mucin that was contained within the goblet cells (Fig. 4, $G$ and $H$ ). The pattern of staining in the wild-type and CF mice was similar with intense staining of the goblet cells at the base of the crypts and strong staining of goblet cells lining the villi. A slight decrease in staining intensity of goblet cells along the villi was commonly seen in the CF animals. Staining with preimmune antisera was negative for goblet cells and positive for the luminal contents, suggesting that the luminal staining with the Muc5ac antiserum was nonspecific (not shown).

Staining with antisera to Muc2, Muc3, and Muc5ac in the colon was also similar when CF mice were compared with their sibling controls. PH497 stained nonglycosylated Muc2 contained within the goblet cells lining the crypts of both the $\mathrm{CF}$ colon (Fig. $4 \mathrm{~K}$ ) and the control colon (Fig. $4 \mathrm{~J}$ ). Fig. $4 \mathrm{M}$ and Fig. $4 N$ are representative of Muc3 staining in the colon of control and CF mice, respectively. Faint staining of the goblet cells lining the crypts is observed in both tissues, with a slight decrease in staining intensity in the CF tissue. As in the CF ileum, the lumen of the CF colon stained intensely for Muc3, whereas staining of the luminal contents with the preimmune antiserum was not as strong (not shown). Again, this may suggest that some of the luminal staining could be specific for Muc3. Specific goblet cell staining with the Muc5ac antiserum was observed at low levels in both control and CF colon (Fig. $4, P$ and $Q)$. Staining of mucus in the lumen was nonspecific as determined by preimmune control antiserum (not shown).

Analysis of Muc1-deficient CF mice. Since Muc1 RNA and protein levels were increased in the GI tract of the CF mice, we continued to examine its role by mating heterozygote $\mathrm{CF}$ mice with Muc1 knockout mice (20) to generate CF mice lack- ing Muc1 (Muc1-deficient CF mice). The rationale was that if Muc1 was a contributing factor, then CF mice lacking it should be healthier. As indicated in Table II, $10 \%$ of the Muc1-deficient CF mice survived until weaning (3.5 wk) as compared with $7 \%$ of the CF mice. Thus, the absence of Muc1 did not significantly affect the survival of the CF mice before weaning at $3.5 \mathrm{wk}$. However, the surviving Muc1-deficient $\mathrm{CF}$ mice were noticeably healthier than the $\mathrm{CF}$ mice upon weaning, with six of eight mice surviving for $>2$ wk on solid food. (A typical CF mouse would not survive $>2-3 \mathrm{~d}$ on a solid diet.) Two of these Muc1-deficient CF animals were killed for histological analyses at 1 and 2 mo of age. At this time they were phenotypically indistinguishable from their littermate controls. Alcian blue staining of multiple levels of ileum and colon revealed no obvious differences between the Muc1-deficient $\mathrm{CF}$ mice and their wild-type littermate controls (not shown). Fig. 5 shows Alcian blue-stained sections of ileum and colon from a Muc1-deficient CF mouse maintained on liquid diet in comparison with a wild-type mouse and a CF mouse on the same diet and a Muc1-deficient mouse. The ileum and colon of the wild-type mouse show staining of goblet cells along the villi and crypts in addition to moderate staining of the luminal contents (Fig. 5, $A$ and $B$ ). As observed previously, the CF mouse shows a marked increase in luminal content staining in the ileum and colon (Fig. 5, $C$ and $D$ ). However, the Muc1-deficient CF mouse shows little to no Alcian blue-positive mucus in either lumen (Fig. 5, $E$ and $F$ ). The Muc1-deficient control mouse (Fig. 5, $G$ and $H$ ) shows Alcian blue staining that is comparable with a wild-type mouse. Thus the luminal mucus obstructions appear to be specific to the CF mouse with Muc1. In total, multiple levels of five sets of tissue have been stained with Alcian blue and PAS; two of the sets of mice were maintained on the liquid diet and three sets of mice were fed solid food. In all cases, luminal content staining in ileum and colon of the Muc1-deficient CF mice was no greater than that observed in wild-type mice on a comparable diet. In addition, Alcian blue staining of Muc1-deficient CF mice on liquid diet was indistinguishable from that of Muc1-deficient CF mice on solid food (not shown). Thus, it appears that a lack of Muc1 greatly decreases the luminal mucus content in the CF mice and as a result the mice appear to be healthier.

Muc2, Muc3, and Muc5ac expression in Muc1-deficient CF mice. It was of interest to determine if there were changes in expression of other mucins in the Muc1-deficient CF mice. No significant differences in Muc2, Muc3, and Muc5ac mRNA expression levels in colon or ileum were detected among $\mathrm{CF}$

Table II. Comparison of 3.5-wk Survival Rates between CF Mice and Muc1-deficient CF Mice

\begin{tabular}{cccc}
\hline CF genotype & Muc1 genotype & 3.5-wk survival & Percentage of total \\
\hline$+/+$ & $+/+$ & 300 & $31 \%$ \\
$+/-$ & $+/+$ & 585 & $61 \%$ \\
$-/-$ & $+/+$ & 69 & $7 \%$ \\
$+/+$ & $-/-$ & 50 & $26 \%$ \\
$+/-$ & $-/-$ & 124 & $64 \%$ \\
$-/-$ & $-/-$ & 20 & $10 \%$ \\
\hline
\end{tabular}

The 3.5-wk survival rate is the number of mice with a corresponding genotype that survived and were weaned. 


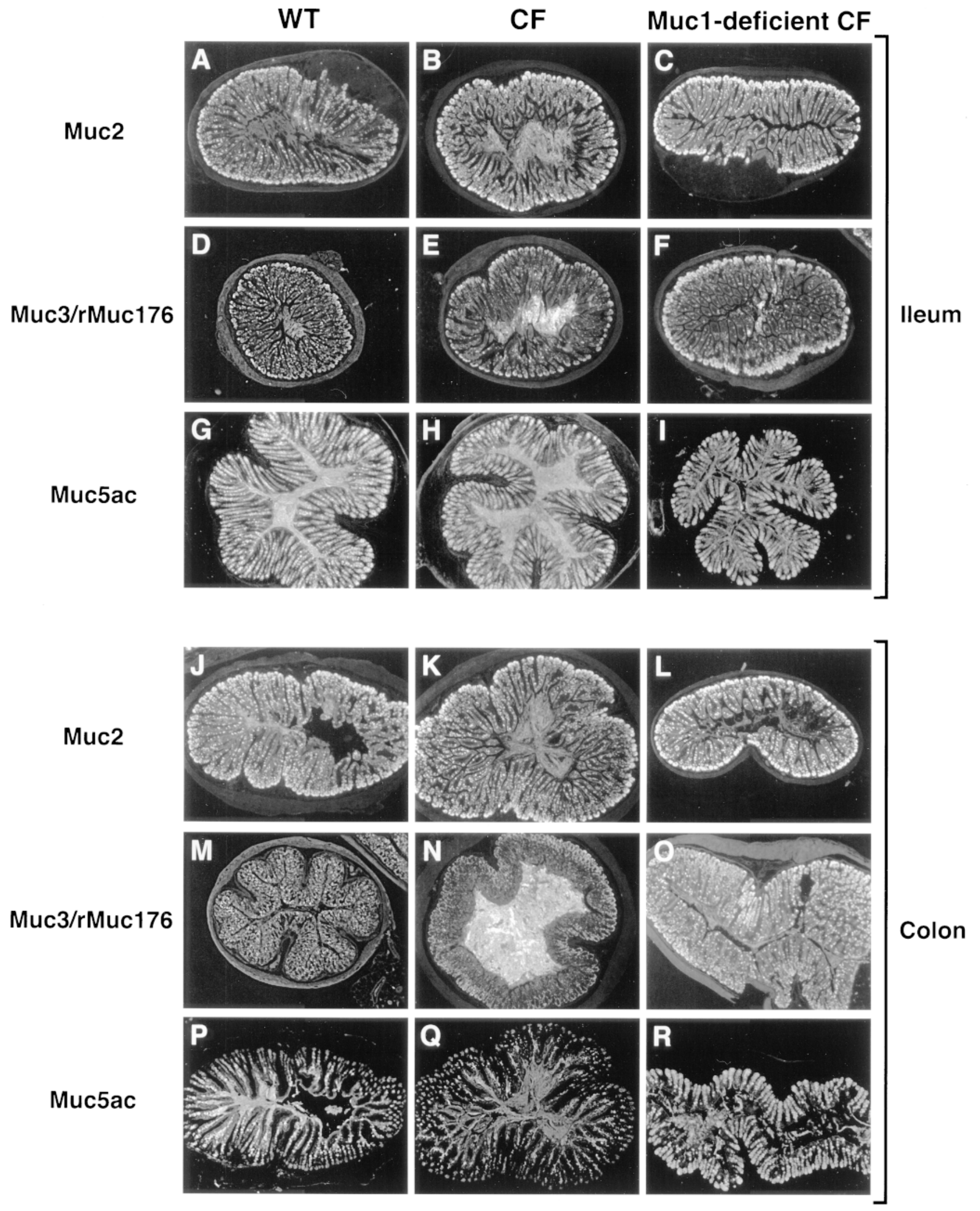

Figure 4. Protein expression of Muc2, Muc3, and Muc5ac in the intestine of CF, Muc1-deficient CF, and control mice. Ileal tissues are shown in $A-I$ and colonic tissues in $J-R$. Wild-type mice are shown in the left column (WT), CF mice in the middle column, and Muc1-deficient CF mice in the right column. Sections stained for Muc2 are $A-C$ and $J-L$, Muc3 are $D-F$ and $M-O$, and Muc5ac are $G-I$ and $P-R$. Mice from which the above sections were taken were all maintained on a liquid diet and were 3-6 mo of age. All rows are comparisons of age-matched animals. These data are representative of five sets of staining. All staining of goblet cells was negative and staining of luminal contents was positive with the ap- 

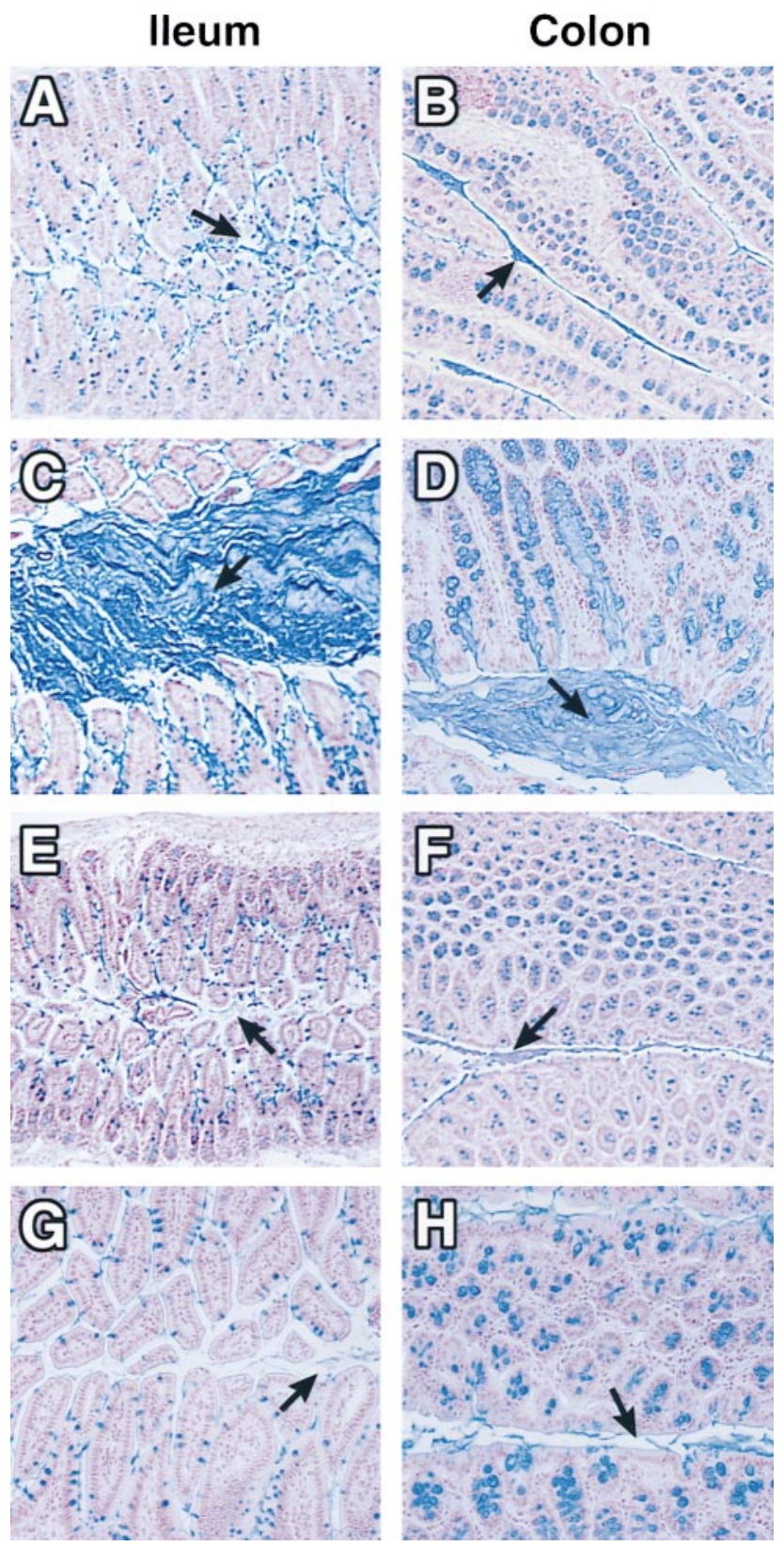

Figure 5. Muc1-deficient CF mice show absence of mucus-filled intestinal lumens. Sections of ileum (left column) and colon (right column) of a wild-type mouse ( $A$ and $B$ ), a CF mouse ( $C$ and $D$ ), a Muc1-deficient CF mouse ( $E$ and $F$ ), and a Muc1-deficient mouse ( $G$ and $H$ ) were stained with Alcian blue in order to visualize mucin glycoproteins. Arrows designate lumens in each of the tissue sections. As expected, the lumens of the CF ileum $(C)$ and the CF colon $(D)$ were distended and filled with Alcian blue-positive mucus. The Muc1-deficient CF mouse ( $E$ and $F$ ) showed lumens free of excess mucus. mice, Muc1-deficient CF mice, and wild-type mice (not shown). Although we recognize that immunohistochemistry is not quantitative, especially using antisera that detect mainly the underglycosylated precursor forms of these secreted mucins, we stained multiple levels of ileum and colon to determine if there were any obvious alterations in protein expression. Both Muc2 and Muc3 exhibited somewhat higher expression levels in the crypts of the ileum in the Muc1-deficient CF mice (Fig. 4, $C$ and $F$ ). This, along with a lack of luminal mucus, may suggest an increase in nonglycosylated Muc2 and Muc3 in the Muc1-deficient mice. At a higher magnification, the goblet cells in the crypts as well as lining the villi of the Muc1-deficient animals appeared distended when compared with the CF mice at a higher magnification (not shown). Staining of Muc5ac appeared to be slightly decreased in intensity in the ileum of the Muc1-deficient CF mice (Fig. $4 I$ ). Again, luminal (background) staining of the Muc1-deficient $\mathrm{CF}$ animals was absent, further indicating the absence of luminal mucus in these mice.

In multiple colonic sections, all three secreted mucins appeared to stain more intensely in the Muc1-deficient CF mice. Antisera to Muc2 and Muc3 showed an increase in goblet cell staining (Fig. 4, $L$ and $O$ ). Specific staining with Muc5ac in the Muc1-deficient $\mathrm{CF}$ colon also appeared to be increased (Fig. 4 $R)$. As in the ileum, luminal content staining with all three antisera was greatly decreased in the Muc1-deficient CF animals. At a higher magnification with all of the antisera, goblet cells in the Muc1-deficient CF mice appeared distended when compared with the CF mice (not shown).

\section{Discussion}

$\mathrm{CF}$ has long been established as a disease involving excessive mucus accumulation. In fact, an early name used to describe the condition was mucoviscidosis. Although the term hypersecretion is frequently used, it is not clear if the pathogenesis of $\mathrm{CF}$ involves mucus hypersecretion, mucus accumulation, or both. Others have shown that an early stage in the development of a hypersecretory epithelium is increased production of mucin RNA $(25,26)$. These works concluded that steady state mucin gene transcription increases in the presence of infection and inflammation, since exposing rat airways to endotoxin (25) and to Sendai virus and $\mathrm{SO}_{2}(26)$ resulted in an increase in specific mucin RNA levels. At the outset of our studies we hypothesized that mucus was hypersecreted in $\mathrm{CF}$, and that because mucins are the major structural component of mucus, they would be hypersecreted as well.

It was surprising that the only mucin to show a significant increase in RNA and protein levels in the CF colon was Muc1, a transmembrane mucin that was not thought to be a major component in mucus gels. Even more striking was the near total loss of PAS- and Alcian blue-positive secretions in the colon and ileum of the Muc1-deficient CF mice. In contrast to the CF mice, many of the Muc1-deficient CF animals were not noticeably different in appearance from their wild-type littermates. As such, many were placed on a solid diet at weaning

Figure 4 legend (Continued)

propriate preimmune control antisera (not shown), unless otherwise stated. Images were captured digitally using identical camera settings and exposure times. All tissues are shown at 40-fold magnification in order that the entire cross-section may be observed and compared. 
before genotyping identified them as CF mice. A small number of these animals survived to adulthood on the solid diet. These animals were ultimately killed for experimental purposes and not because they appeared in ill health. A larger number of Muc1-deficient CF animals that were initially placed on to solid food did necessitate a switch to the liquid diet due to ill health and an inability to thrive. These Muc1deficient $\mathrm{CF}$ mice did recover after being placed on the liquid diet. In contrast, it was extremely rare when a sickly CF animal recovered, even after weaning directly on to the liquid diet. It is interesting that the role of Muc1 in CF appears to be important in the adult animals since an increased survival rate in Muc1-deficient animals was observed only in the adults. This correlates with the increases in Muc1 RNA that were observed only in the adult CF mice and not in the weanlings.

The easiest explanation for the decrease in Alcian blue/ PAS-positive material in the lumens of the ileum and colon of the Muc1-deficient CF animals is that it was Muc1 that was being secreted. Although Muc1 is commonly considered a membrane-associated mucin, secreted forms have been described frequently. Secreted MUC1 is found at high levels in human breast milk and in human bile $(12,27)$. MUC1 has also been detected in serum from patients with breast and pancreatic cancer $(10,11)$ and secreted forms of MUC1 have been shown to exist in tissue culture (13-15). Mouse cells, when transfected with the full-length MUC1 cDNA, secrete MUC1 protein lacking the cytoplasmic tail into the culture medium (13). In addition, the colon carcinoma cell line, COLO 205, produces MUC1 (H-CanAg) that is found in the cell culture medium $(14,15)$. Moreover, MUC1 derived from this cell line was shown to be heavily glycosylated ( $85 \%$ of its molecular mass of $800,000 \mathrm{D}$ due to carbohydrate) with average sugar chain lengths of 15 residues. Fucose and sialic acid were abundantly expressed at two to three residues per chain for each sugar (14). MUC1 isolated from bile is even larger and more heavily glycosylated (12). In these cases, MUC1 has many of the characteristics described for the classical secretory mucins, lacking mainly disulfide linkages. The presence of a cleavage site at which MUC1 protein is cleaved in the endoplasmic reticulum resulting in a protein lacking a transmembrane domain and a cytoplasmic tail has also been described (28). Therefore, it is not unreasonable to postulate that Muc1 may make a significant contribution to the physical properties of the mucus gel.

At this time, we are not able to determine if Muc1 is a major component of the luminal secretions in the CF mice. The existence of this secreted form is difficult to detect in mouse, as antisera to the extracellular portion of Muc1 are unavailable. Since our current antibody, CT1, recognizes the transmembrane/cytoplasmic tail region, it is not suitable for detecting secreted Muc1. As a result, presently we are generating CF mice with human MUC1 in place of mouse Muc1. Provided that MUC1 glycosylation in the colon is not altered significantly, we have reagents to the human extracellular domain that should enable us to determine if MUC1 is a major component of the mucus obstructions.

The decrease in RNA levels of Muc2, Muc3, and Muc5ac in the $\mathrm{CF}$ mice remains unexplained. At this point, we cannot rule out the possibility that the intestinal obstructions that are observed in the CF mouse are due to the secretion of Muc2, Muc3, or Muc5ac. As mentioned earlier, the antisera that were used to detect these mucins stain only the immature or intracellular forms. Even though the goblet cell-containing levels of these mucins remained unchanged between the $\mathrm{CF}$ mice and their sibling controls, these data tell us nothing about the secreted levels of the mature, glycosylated molecules. Although not conclusive, the increased background staining in the lumens of the $\mathrm{CF}$ animals with Muc3 may suggest further study of this glycoprotein. As such, another possible explanation for the decrease in Alcian blue/PAS-positive material in the lumens of the ileum and colon of the Muc1-deficient CF animals could be that Muc1 is affecting the release of other secreted mucins by an as yet unexplained mechanism. It is possible that the transmembrane mucin Muc1 acts as a tether for the mucus gel formed by the cysteine-rich mucins. Without Muc1 on the surface of the epithelium, the mucus gel may not be readily retained in the lumen of the GI tract, accounting for the lack of excess mucus accumulation in Muc1-deficient $\mathrm{CF}$ animals. Immunohistochemical results using antisera to nonglycosylated Muc2, Muc3, and native Muc5ac revealed moderate increases in staining intensity of the goblet cells in the ileum and colon of the Muc1-deficient CF animals. In addition, the lumens of these animals were completely free of excess mucus accumulation as indicated by a lack of Alcian blue/PAS staining and of luminal background staining with any of the above antisera. Thus, it may be reasonable to speculate that Muc1 upregulates the release of secreted mucins in the $\mathrm{CF}$ mice and, in its absence, the Muc1-deficient CF mice have decreased mucin release. In addition, goblet cells in the wild-type mice and in the Muc1-deficient mice appeared distended when compared with the CF mice. This may also suggest that the mucus accumulation observed only in the CF animals is a result of goblet cell release. Aside from Muc2, Muc3, and Muc5ac, these secreted mucins may just as well be "new" unidentified mucins. To examine these possibilities, we are in the process of isolating the mucus from the $\mathrm{CF}$ mouse intestine in order to biochemically purify and subsequently identify the mucins that are present.

\section{Acknowledgments}

The authors thank Dr. Beverly Koller for kindly providing breeding pairs for generating the CF mice. We also thank Drs. Jim Gum and Young Kim for providing the Rib-3 antiserum, Dr. Gunnar Hansson for sharing the Muc2 antiserum, and Dr. Sam Ho for providing the antiserum to Muc5ac. We are grateful to Anita Jennings and Karen LaCombe for their expert histological assistance, to Marv Ruona and Julie Jensen for their assistance with the graphics, and to Amy Weaver for her help with the statistical analyses.

This study was funded by the National Institutes of Health, Specialized Centers of Research DK49184 (to S.J. Gendler) and Research Fellowship Award DK09264 (to R.R. Parmley); and the Cystic Fibrosis Foundation, GENDLE97Z0 (to S.J. Gendler).

\section{References}

1. Samet, J.M., and P.W. Cheng. 1994. The role of airway mucus in pulmonary toxicology. Environ. Health Perspect. 102:89-103.

2. Gendler, S.J., and A.P. Spicer. 1995. Epithelial mucin genes. Annu. Rev. Physiol. 57:607-634.

3. Rogers, D.F. 1994. Airway goblet cells: responsive and adaptable frontline defenders. Eur. Respir. J. 7:1690-1706.

4. Koller, B.H., H.-S. Kim, A.M. Latour, K. Brigman, R.C. Boucher, P. Scambler, B. Wainwright, and O. Smithies. 1991. Toward an animal model of cystic fibrosis: targeted interruption of exon 10 of the cystic fibrosis transmembrane regulator gene in embryonic stem cells. Proc. Natl. Acad. Sci. USA. 88: 10730-10734. 
5. Snouwaert, J.N., K.K. Brigman, A.M. Latour, N.N. Malouf, R.C. Boucher, O. Smithies, and B.H. Koller. 1992. An animal model for cystic fibrosis made by gene targeting. Science. 257:1083-1088.

6. Mearns, M.B. 1993. Cystic fibrosis: the first 50 years. A review of the clinical problems and their management. In Cystic Fibrosis Current Topics. J.A. Dodge, D.J.H. Brock, and J.H. Widdicombe, editors. John Wiley \& Sons Ltd., New York. 217-250.

7. Pemberton, L., J. Taylor-Papadimitriou, and S.J. Gendler. 1992. Antibodies to the cytoplasmic domain of the MUC1 mucin show conservation throughout mammals. Biochem. Biophys. Res. Commun. 185:167-175.

8. Gendler, S.J., C.A. Lancaster, J. Taylor-Papadimitriou, T. Duhig, N. Peat, J. Burchell, L. Pemberton, E.-N. Lalani, and D. Wilson. 1990. Molecular cloning and expression of human tumor-associated polymorphic epithelial mucin. J. Biol. Chem. 265:15286-15293.

9. Spicer, A.P., G. Parry, S. Patton, and S.J. Gendler. 1991. Molecular cloning and analysis of the mouse homologue of the tumor-associated mucin, MUC1, reveals conservation of potential O-glycosylation sites, transmembrane, and cytoplasmic domains and a loss of minisatellite-like polymorphism. J. Biol. Chem. 266:15099-15109.

10. Burchell, J., D. Wang, and J. Taylor-Papadimitriou. 1984. Detection of the tumor associated antigens recognised by the monoclonal antibodies HMFG-1 and 2 in serum from patients with breast cancer. Int. J. Cancer. 34: $763-768$.

11. Metzgar, R.S., N. Rodriguez, O.J. Finn, M.S. Lan, V.S. Daasch, P.D. Fernsten, W.C. Meyers, W.S. Sindelar, R.S. Sandler, and H.F. Siegler. 1984. Detection of a pancreatic cancer-associated antigen (DU-PAN-2 antigen) in serum and ascites of patients with adenocarcinoma. Proc. Natl. Acad. Sci. USA. 81: $5242-5246$.

12. Baeckström, D., N. Karlsson, and G.C. Hansson. 1994. Purification and characterization of sialyl-Le ${ }^{\mathrm{a}}$-carrying mucins of human bile. Evidence for the presence of MUC1 and MUC3 apoproteins. J. Biol. Chem. 269:14430-14437.

13. Boshell, M., E.-N. Lalani, L. Pemberton, J. Burchell, S.J. Gendler, and J. Taylor-Papadimitriou. 1992. The product of the human MUC1 gene when secreted by mouse cells transfected with the full-length cDNA lacks the cytoplasmic tail. Biochem. Biophys. Res. Commun. 185:1-8.

14. Baeckstrom, D., G.C. Hansson, O. Nilsson, C. Johansson, S.J. Gendler, and L. Lindholm. 1991. Purification and characterization of a membrane-bound and a secreted mucin-type glycoprotein carrying the carcinoma-associated sialyl-Le ${ }^{a}$ epitope on distinct core proteins. J. Biol. Chem. 266:21537-21547.

15. Zhang, K., D. Baeckstrom, H. Breving, and G.C. Hansson. 1996. Secreted MUC1 mucins lacking their cytoplasmic part and carrying sialyl-Lewis a and $\mathrm{x}$ epitopes from a tumor cell line and sera of colon carcinoma patients can inhibit HL-60 leukocyte adhesion to E selectin-expressing endothelial cells. J. Cell. Biochem. 60:538-549.

16. Gonqiao, X., L.-J. Huan, I.A. Khatri, D. Wang, A. Bennick, E.F Raafat, G.G. Forstner, and J.F. Forstner. 1992. cDNA for the carboxyl-terminal region of a rat intestinal mucin-like peptide. J. Biol. Chem. 267:5401-5407.

17. Khatri, I.A., G.G. Forstner, and J.F. Forstner. 1993. Suggestive evidence for two different mucin genes in rat intestine. Biochem. J. 294:391-399.

18. Gum, J.R., J.W. Hicks, R.E. Lagace, J.C. Byrd, N.W. Toribara, B. Siddiki, F.J. Fearney, D.T. Lamport, and Y.S. Kim. 1991. Molecular cloning of rat intestinal mucin. J. Biol. Chem. 266:22733-22738.

19. Shekels, L.L., C. Lyftogt, M. Kieliszewski, J.D. Filie, C.A. Kozak, and S.B. Ho. 1995. Mouse gastric mucin: cloning and chromosomal localization. Biochem. J. 311:775-785.

20. Spicer, A.P., G.J. Rowse, T.K. Lidner, and S.J. Gendler. 1995. Delayed mammary tumor progression in Muc1 null mice. J. Biol. Chem. 270:3009330101.

21. Alonso, S., A. Minty, Y. Bourlet, and M.J. Buckingham. 1986. Comparison of three actin-coding sequences in the mouse: evolutionary relationships between the actin genes of warm-blooded vertebrates. Mol. Evol. 23:11-22.

22. Cook, H.C. 1990. Carbohydrates. In Theory and Practice of Histological

Techniques. J.D. Bancroft and A. Stevens, editors. Churchill Livingstone, New York. 177-213.

23. Pemberton, L., J. Taylor-Papadimitriou, and S.J. Gendler. 1992. Antibodies to the cytoplasmic tail domain of the MUC1 mucin show conservation throughout mammals. Biochem. Biophys. Res. Commun. 85:167-175.

24. Karlsson, N.G., M.E. Johansson, N. Asker, H. Karlsson, S.J. Gendler, I. Carlstedt, and G.C. Hansson. 1996. Molecular characterization of the large heavily glycosylated domain glycopeptide from the rat small intestinal Muc2 mucin. Glycoconj. J. 13:823-831.

25. Steiger, D., J. Hotchkiss, L. Bajal, J. Harkema, and C. Basbaum. 1995. Concurrent increased in the storage and release of mucin-like molecules by rat airway epithelial cells in response to bacterial endotoxin. Am. J. Respir. Cell. Mol. Biol. 12:307-314.

26. Jany, B., M. Gallup, T. Tsuda, and C. Basbaum. 1991. Mucin gene expression in rat airways following infection and irritation. Biochem. Biophys. Res. Commun. 181:1-8.

27. Patton, S., S.J. Gendler, and A.P. Spicer. 1995. The epithelial mucin MUC1, of milk, mammary gland and other tissues. Biochim. Biophys. Acta. 1241:407-424.

28. Ligtenberg, M.J.L., L. Kruijshaar, F. Buijs, M. van Meijer, S.V. Litvinov, and J. Hilkens. 1992. Cell-associated episialin is a complex containing two proteins derived from a common precursor. J. Biol. Chem. 267:6171-6177. 